\title{
APLICAÇÃO DE TEORIA DAS FILAS PARA MELHORIA DO ATENDIMENTO EM UMA INSTITUIÇÃO FINANCEIRA DE SALGUEIRO-PE
}

\author{
Eugênio Jose de Souza Neto (Universidade de Pernambuco) eugenio.neto@santander.com.br \\ Danillo Rodrigues Silva Bento Oliveira (UNIVASF) danillo.oliveira@univasf.edu.br \\ Éverton Crístian Rodrigues de Souza (UNIVASF) everton.souza@univasf.edu.br \\ Lívia Jordana Gois e Silva Patriota (UNIVERSIDADE DE PERNAMBUCO) liviajordanap@gmail.com \\ Maria Fernanda Ângelo do Carmo (UNIVASF) fernanda.angelo@ discente.univasf.edu.br
}

\section{Resumo}

A falta de planejamento e gerenciamento do atendimento pessoal nos caixas bancários prejudicam tanto os clientes, por não gostarem de esperar em filas, quanto os bancos, que podem vir a sofrer sanções ou multas, conforme a Lei $\mathrm{n}^{\circ}$ 12.264/2002, afetando negativamente a imagem da instituição. Nesse contexto, esta pesquisa tem como objetivo analisar a aplicação da teoria das filas a fim de melhorar a qualidade de atendimento aos clientes de uma instituição financeira. Classifica-se como quantitativa, de natureza aplicada, e de caráter descritivo, adotando como procedimento a realização de um estudo de caso, na busca de compreender melhor os acontecimentos ocorridos (CERVO, 2002; YIN, 2005). Para análise dos dados fez-se o uso de um software de simulação, o ProdModel, além do tratamento analítico dos dados. A instituição possui quatro pontos de atendimento, entre eles, optou-se pela análise e estudo dos Caixas (de atendimento pessoal). Assim, foi realizado o levantamento de informações quantitativas do tempo dos clientes na fila e tempo de atendimento dos usuários, em um período considerado de pico, em relação à fila preferencial e à fila de atendimento ao público em geral. Os resultados apontam a proposta de melhoria mediante unificação do atendimento como a mais viável, resultado em atendimento mais ágil com menor dispêndio de recursos.

Palavras-Chaves: (Setor bancário. Qualidade no atendimento. Teoria das filas)

\section{Introdução}

Segundo a Febraban (2017), o setor bancário brasileiro é conhecido internacionalmente como um dos setores que mais investe em tecnologia da informação, buscando o aperfeiçoamento do sistema. Com a ampliação de novas tecnologias, advindas da evolução das técnicas de produção e investimentos na área, atualmente no serviço bancário os clientes dispõem de canais facilitadores como os caixas eletrônicos, aplicativos para celular, internet banking e central de atendimento, obtendo serviços ou produtos com horários flexíveis, que funcionam $24 \mathrm{~h}$ por dia.

Conforme pesquisa realizada em 2016, no Brasil, uma em cada cinco transações com movimentação financeira é feita via Internet Banking ou Mobile Banking (FEBRABAN, 2017). Tal resultado demonstra que as agências físicas passaram a ter um papel mais consultivo, por boa parte dos clientes, mostrando a evidente migração para os canais de autoatendimento. 
No entanto, mesmo diante da existência de tais canais, ainda há uma grande parcela de clientes que preferem realizar suas operações de maneira tradicional, ou seja, dirigindo-se a agência bancária. Com isso, essa área operacional do banco precisa atender essa demanda de forma competente, a fim de evitar insatisfações, como as proporcionadas pela espera em filas. A Lei Estadual $n^{\circ} 12.264 / 2002$, ordena que a duração máxima de espera nas filas dos bancos não deve ir além dos 15 minutos, podendo chegar a 30 minutos nos dias de pico, podendo o excedente causar sanções e multas às instituições financeiras (PERNAMBUCO, 2002).

Visando satisfazer, ou até mesmo superar, a expectativa do cliente, se faz necessário planejar a capacidade de atendimento da demanda, tratando-se com o rigor que a abordagem científica proporciona. Logo, a Teoria das Filas se aplica à resolução de problemas desta natureza, como abordam LIMA; ANHOLON; RAMPASSO; QUELHAS (2016), que apontam a necessidade crescente de conquistar clientes e mantê-los fiéis, destacando a aplicação da consagrada Teoria das Filas na tomada de decisões nas agências bancárias com a finalidade de apontar oportunidades de melhorias no tempo de espera dos clientes.

Embora o dinheiro seja a matéria prima de uma instituição bancária, na escolha por uma instituição financeira em detrimento de outra, muitos clientes consideram de suma importância a qualidade do atendimento ao analisarem os pontos, a fim de centralizar suas operações financeiras em um banco.

Segundo Slack (2013), em todas as operações a qualidade é a parte mais perceptível e, também, um dos objetivos mais almejados. Tendo em vista que é mais facilmente identificada pelo cliente no front office, a qualidade é mais utilizada pelos clientes na avaliação da operação.

Partindo da premissa de que o capital esteja disponível, uma das variantes mais importantes para análise da qualidade dos serviços é o atendimento, feito que muitas vezes direciona a satisfação dos consumidores de um banco e sua respectiva reputação perante o mercado. Especificamente, um dos principais critérios de julgamento pelo cliente é o tempo de espera nas filas para atendimento.

As instituições financeiras vêm desenvolvendo através da tecnologia, métodos para evitar a espera nas filas, tendo como consequência mais um indicador de bons serviços, que por sua vez, conquista ainda mais o mercado em face da satisfação da clientela. É o que afirma a Febraban (2017), quando diz que a disseminação de novas tecnologias que facilitam a comunicação interna de colaboradores e reduzem dramaticamente o tempo de processos 
burocráticos, tornará os grandes bancos de varejo mais rápidos, eficientes e com menor custo operacional.

Segundo Hillier (2006), o tempo que a população de um país perde em filas é um importante fator tanto na qualidade de vida nesse país quanto na eficiência da economia dessa nação.

A Pesquisa Operacional utiliza modelos matemáticos e ou lógicos para solucionar problemas reais, apresentando uma alta multidisciplinaridade. Normalmente, essas soluções são encontradas apenas por alguns dos algoritmos. Desde o endereço da linha e modelos de simulação no estudo de filas.. (SANTOS; QUINTAL; PAIXÃO; GOMES, 2015)

Baseado nisso, consegue-se identificar que o atendimento aos clientes pode proporcionar não somente uma satisfação perante a instituição, mas também até condicionar um bem-estar em sua vida, e, sem dúvidas, mobilizar recursos para outros fins mais produtivos.

Voltando ao contexto brasileiro, segundo informações do Banco Central do Brasil (2017), as cinco instituições bancárias brasileiras com maior índice de reclamações no período de janeiro a fevereiro de 2017 são: Bradesco, Banco do Brasil, Caixa Econômica, Santander e Itaú, como mostram o Quadro 1. Logo, é evidente que torna-se relevante a abordagem do problema de filas em instituições financeiras mediante tratamento científico do contexto empírico.

Quadro 1 - Ranking das Instituições por índice de reclamações (Jan e Fev/2017)

\begin{tabular}{|c|c|c|}
\hline Posição & Instituição Financeira & $N^{\circ}$ de Reclamações Reguladas Procedentes \\
\hline $1^{\circ}$ & Bradesco & 1598 \\
\hline $2^{\circ}$ & Banco do Brasil & 1024 \\
\hline $3^{\circ}$ & Caixa Econômica & 1286 \\
\hline $4^{\circ}$ & Santander & 514 \\
\hline $5^{\circ}$ & Itaú & 790 \\
\hline & TOTAL DE RECLAMAÇÕES & $\underline{5212}$ \\
\hline
\end{tabular}

Fonte: Elaboração própria (2017), com base em Banco Central do Brasil (2017).

Segundo Slack (2013, p. 183), "a gestão dos sistemas de fila, geralmente envolve tentar administrar as percepções e expectativas dos clientes de alguma forma". O setor de serviços está à procura de índices que tenham eficácia no processo de atendimento ao cliente diante do cenário de mercado vigente. Para trabalhar de modo acirrado em economias com a competitividade cada vez mais alta, as organizações precisam estruturar os esforços de seus distintos setores e áreas. 
Dessa forma, esta pesquisa tem a finalidade de resolver o seguinte problema: Como melhorar a satisfação de usuários e clientes no tocante à qualidade do atendimento nos caixas de uma agência bancária na cidade de Salgueiro-PE?

Ante essa problemática, a pesquisa objetiva analisar como a aplicação da teoria das filas pode melhorar a qualidade do atendimento aos clientes de uma instituição financeira. Para tanto, os seguintes marcadores serão alcançados à guisa de objetivos específicos: Descrever o modelo de filas atual da área de atendimento dos caixas; Aplicar a teoria das filas para redução da insatisfação com o tempo de espera dos clientes da instituição; Propor melhorias para atendimento da demanda dos clientes com base nos resultados alcançados. Nesse contexto, os eixos temáticos relativos à Qualidade e Teoria das filas são fundamentais para conferir base teórica na abordagem do problema de pesquisa enunciado. As seções seguintes lançam mão desses fundamentos teóricos.

\section{Fundamentação teórica}

\subsection{Qualidade}

Segundo Slack (2008), qualidade é não cometer erros e satisfazer a seus consumidores, fornecendo bens e serviços isentos de erro, "adequados a seus propósitos". A qualidade é definida do ponto de vista do cliente como aquilo que atenda às suas necessidades e expectativas quanto às características do produto ou na prestação de serviços (CORRÊA; CORRÊA, 2007).

Como fator competitivo, a qualidade deve ser projetada a fim de atender a finalidade estratégica da operação, ou seja, é necessário sempre perceber as expectativas e percepções dos consumidores em relação aos serviços prestados. Um dos grandes desafios das organizações é ofertar serviços de qualidade para seus clientes, sejam eles internos ou externos. Os clientes internos são os colaboradores da própria empresa, e os externos são aqueles que adquirem os serviços.

Segundo Martins e Laugeni (2005), os elementos da qualidade de serviços são: confiabilidade, cortesia, comunicação, capacidade para entender as necessidades do cliente, fácil utilização, credibilidade, ser competente, segurança, rapidez na resposta e aspectos visíveis. 
Do ponto de vista de Slack (2002), a qualidade em uma operação pode ser vista com base em três parâmetros: conformidade, consistência e expectativas. A conformidade acontece quando o serviço atende as especificações, de acordo com a necessidade de seus clientes. É consistente quando os materiais, instalações e processos garantem as especificações do serviço dentro do projeto. Já as expectativas dizem respeito a quando o serviço atende a expectativa dos seus consumidores, de acordo com o pacote de valor gerado para o usuário. Incluem-se dentro das expectativas, as necessidades e exigências dos consumidores:

- Exigência: a organização proporciona aquilo que o consumidor deseja;

- Necessidades: quando a organização atende aos requisitos básicos do consumidor.

Os três parâmetros citados por Slack (2002) serão utilizados para averiguar a qualidade no atendimento, servindo como base para analisar os tempos e dados da simulação.

No caso da prestação de serviços, a presença do cliente é mais intensa e, nesse caso, a qualidade no atendimento deve ser mais acentuada e deve-se gerir os processos com base na capacidade de atendimento e demanda de clientes. No que toca à adequação da capacidade de produção do serviço à demanda, a Teoria das filas pode colocar bastante luz nas decisões sobre como melhor envidar esforços e recursos, e por isso é abordado em seguida.

\subsection{Teoria das filas}

Segundo Gomes et al. (2016), as filas já fazem parte do cotidiano da nossa sociedade, de forma que sempre há pessoas em alguma fila, seja no cinema, no supermercado ou no serviço postal. Ainda segundo os autores, as pessoas terminam por se acostumar, de certa forma, com esse processo de espera. Porém, o tempo perdido nas filas gera transtornos para as pessoas, para as empresas e enaltece o mau funcionamento desse sistema.

As filas se formam quando a demanda por um determinado serviço é maior que a oferta proporcionada. Para que haja um equilíbrio entre a satisfação do cliente e a prestação do serviço de forma econômica, os pesquisadores lançam mão de métodos e fórmulas matemáticas para este fim (GOMES et al., 2016).

Nas primeiras décadas do século XX, em Copenhagen, Dinamarca, o matemático A. K. Erlang desenvolveu uma série de fórmulas matemáticas para estudar os problemas dos congestionamentos de chamadas em centrais telefônicas. Em 1917, Erlang propõe uma teoria para o tráfego de ligações, na qual conseguia determinar a probabilidade de diferentes 
números de ligações estarem aguardando para serem atendidos, e o tempo médio com maior probabilidade de espera quando o sistema estivesse equilibrado. Dessa forma, teve origem a Teoria das filas, sendo a partir deste marco histórico, posto em desenvolvimento ao longo das décadas seguintes. As técnicas matemáticas mais estudadas e usuais na resolução de problemas são as que envolvem o gerenciamento dos tempos de atendimento e dimensionamento de estações de trabalho.

Segundo Slack (2008), praticamente, todas as organizações que processam fluxos (quer sejam fluxos de pessoas, materiais ou de informações) e que estão sujeitas a alguma restrição de capacidade de recursos, encaram de uma forma ou de outra, o problema de filas de espera.

A teoria das filas é uma técnica analítica que utiliza modelos matemáticos para estudar os parâmetros de uma fila de um sistema real. Os parâmetros são: tempo médio de espera, tamanho médio da fila, taxa média de utilização do servidor. A teoria das filas possibilita descobrir a melhor forma de atender o cliente prestando o serviço de forma econômica (SAMPAIO; OLIVEIRA, 2013). A Figura 1 é uma demonstração visual de um sistema de filas na prática.

\section{Figura 1 - Representação de um sistema de filas}

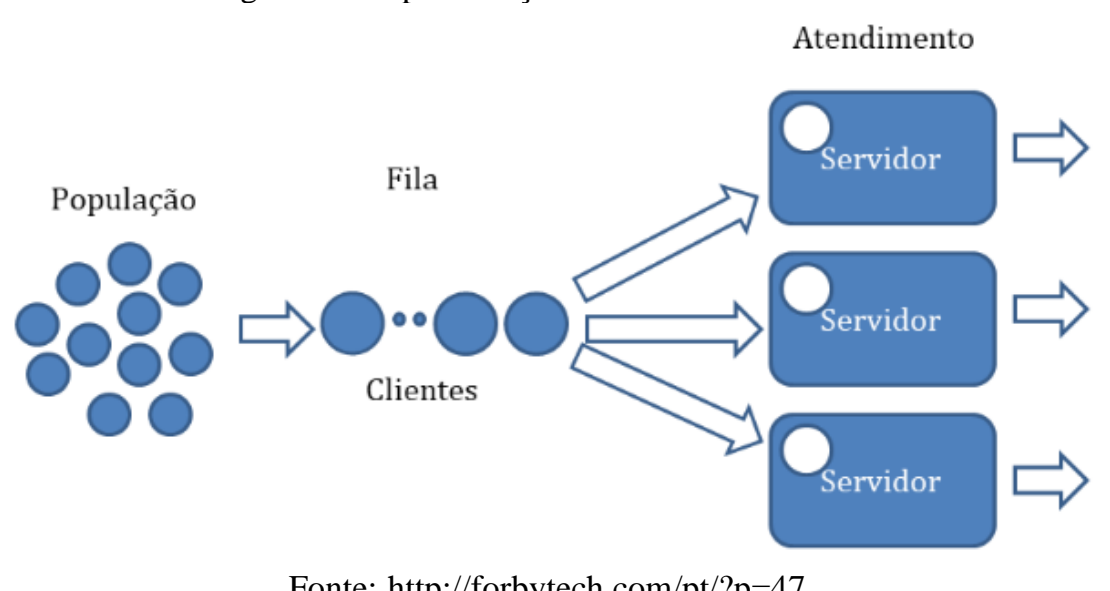

Fonte: http://forbytech.com/pt/?p=47

Segundo Sampaio e Oliveira (2013), no sistema de filas o cliente entra para receber a prestação de serviço que comprou ou necessita. Logo, a situação da fila se torna indispensável, pois existem outros clientes com necessidades semelhantes e os funcionários disponíveis não são capazes de atender a todos simultaneamente. Por fim, o cliente passa pela fila é atendido e, finalmente, sai do sistema.

\subsubsection{Características do sistema de filas}


Uma população é um conjunto de clientes. Esta população pode ser finita ou infinita. Na ótica de Corrêa (2008), população finita trata-se de sistemas cuja população-fonte é pequena e fica relevantemente afetada pelos clientes que estão sendo servidos. Em contrapartida, uma população infinita, conforme o mesmo autor, versa sobre uma população grande o suficiente para não ser afetada pela "saída" de um cliente que entre no sistema de filas.

De acordo com Slack (2013, p; 183), uma fonte de clientes infinita assume que exista grande número de clientes potenciais de modo que há sempre a possibilidade de outro cliente chegar, independentemente de quantos estejam sendo servidos. A maioria dos sistemas de filas que lidam com mercados externos, possui fontes infinitas de clientes ou próximas ao infinito.

Em um sistema de filas há uma taxa de chegada, que diz respeito à proporção em que os clientes que necessitam serem servidos chegam aos atendentes. Raramente obtêm-se uma taxa constante e previsível. Sendo assim, utiliza-se do tratamento analítico para calcular o tempo médio de atendimento, através da seguinte fórmula:

$\mathrm{T} \mathrm{S}=1 / \mu-\lambda ;$ em que:

$\lambda=$ Taxa média de chegada de clientes ao sistema.

$\mu=$ Taxa média de atendimento de um canal.

Para encontrarmos o tempo médio de espera na fila, utilizamos o cálculo:

$\mathrm{T} \mathrm{F}=\lambda / \mu(\mu-\lambda)$

Destarte, compreendidos tais aspectos teóricos que fundamentam a presente pesquisa, abordaremos na próxima seção determina as características metodológicas que nortearam o estudo para o alcance do objetivo outrora citado.

\section{Aspectos metodológicos}

\subsection{Caracterização da pesquisa}

No que se refere à abordagem, o presente estudo possui caráter de pesquisa quantitativa, visto que as informações foram tratadas para interpretação dos resultados e análise segundo métodos matemáticos e estatísticos que fundamentam o software de simulação utilizado. Quanto à natureza classifica-se como pesquisa aplicada (ROESCH, 2007).

No que concerne aos objetivos, a pesquisa é considerada descritiva, pois descreve as situações com precisão, estabelecendo relações entre as variáveis (CERVO, 2002). No que tange aos 
procedimentos, a pesquisa classifica-se como um estudo de caso, pois busca a compreensão e investigação de acontecimentos que ocorrem no dia a dia, inseridos em um contexto real e de cunho complexo (YIN, 2005, p.19).

\subsection{Instrumentos de coleta e análise de dados}

As informações foram adquiridas através de relatórios disponíveis nos sistemas de gerenciamento de atendimento, disponibilizados pela instituição em estudo, e, também, através da análise observacional. Para realização da análise dos dados coletados fez-se uso do software ProModel, um programa de simulação de fácil acesso, com utilização constante em todo o mundo, tanto pelas universidades como pelas organizações, que dispõe de versão para estudantes, sendo um dos mais populares no Brasil (BATEMAN, 2013; SANTOS FILHO et al., 2014).

Através do ProModel é possível criar programações visuais, onde o fluxo é disposto em forma de blocos, que podem representar objetos, transações ou pessoas, formando um ambiente estabelecido com propriedades e regras, criando espaço para elementos dinâmicos que transcorrem dentro desse ambiente.

Também foi executado o tratamento analítico das situações utilizando fórmulas e cálculos da Teoria das Filas, operacionalizados em planilhas eletrônicas. Sendo assim, foram simulados tanto o modelo de filas vigente como o modelo proposto. A seção subsequente demonstra os resultados da pesquisa.

\section{Análise e discussão dos resultados}

\subsection{Sistema de filas estudado}

A instituição financeira escolhida para análise conta com 13 colaboradores, ambiente agradável, dispõe de composição tradicional, e possui quatro pontos de atendimento, sendo eles: Atendimento Pessoa Física, Atendimento Pessoa Física Alta Renda, Atendimento Pessoa Jurídica e Caixas. O último item foi o escolhido para análise, devido à demanda para atendimento ser maior e nos dias de pico, implicando em risco elevado de reclamações e imputações legais. Nos caixas são realizados os serviços de depósitos, saques, ordens de pagamento, recebimento de boletos, transferência de valores entre contas, entre outros. $\mathrm{Na}$ bateria de caixa dispõe-se de três computadores e dois operadores. 
Para atendimento ao público, a instituição financeira funciona de segunda a sexta, das 10:00 as 15:00, e existem dois tipos de demandas de atendimento no setor de caixas, prioridades por lei e público em geral. $\mathrm{O}$ atendimento das filas é realizado conforme a Figura 2.

Figura 2 - Diagrama do atendimento atual Sistema

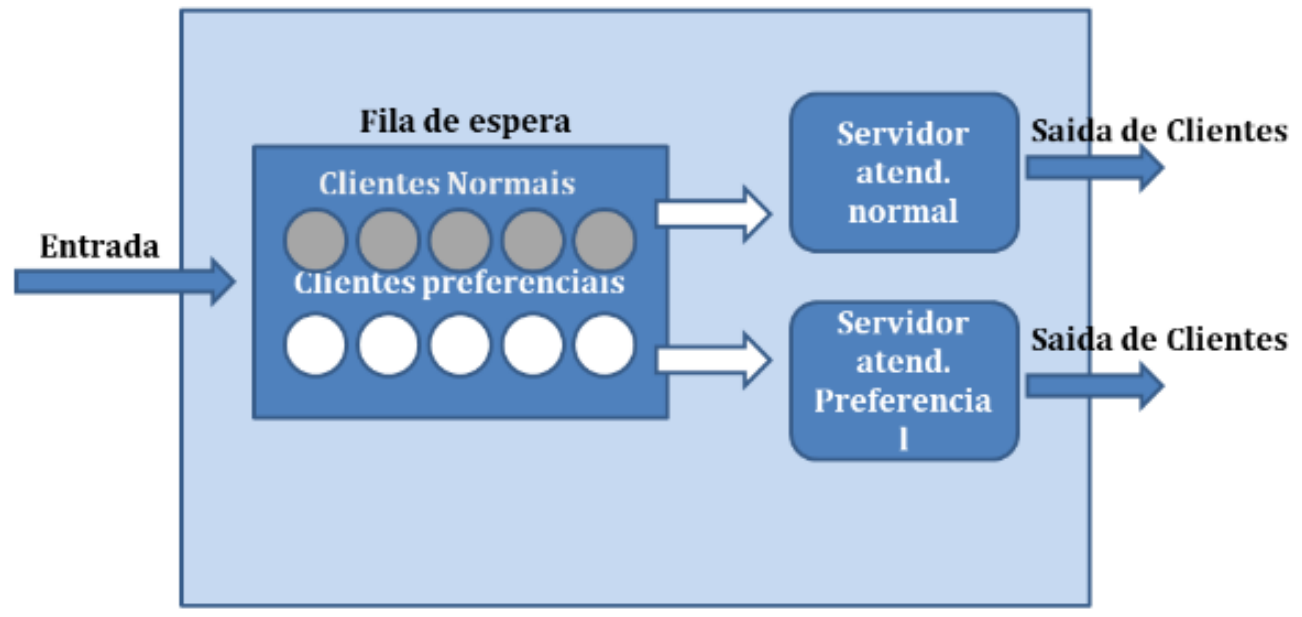

Fonte: Elaboração própria (2020)

\subsection{Simulação do sistema de filas estudado}

O levantamento das informações quantitativas do tempo dos clientes na fila e tempo de atendimento dos usuários foi realizado no dia $06 / 10 / 2017$, período considerado dia de pico, ou seja, grande demanda de pessoas nas instituições financeiras, devido ao recebimento de proventos de vários órgãos.

Conforme a distribuição, a taxa média de chegada $(\lambda)$ é de 86 clientes no caixa preferencial e 166 no caixa normal, a uma taxa média de saída de 72 pessoas no atendimento prioritário e 130 no atendimento em geral, os demais continuavam no sistema ou saíram da fila sem atendimento, após o término do tempo de observação (5 horas).

Durante o período observado, não houve inclusão de mais um operador de caixa, bem como, não houve ausência dos dois operadores que realizavam atendimento. No Quadro 2, são apresentados os resultados de tempo médio de atendimento e tempo médio de espera na fila, obtidos através do tratamento analítico, explanado durante a fundamentação teórica.

\begin{tabular}{cccc}
\hline \multicolumn{4}{c}{ Quadro 2 - Tempo médio de atendimento e de espera } \\
\hline Caixa & $\begin{array}{c}\text { Senhas } \\
\text { atendidas }\end{array}$ & $\begin{array}{c}\text { Tempo médio de } \\
\text { atendimento }\end{array}$ & $\begin{array}{c}\text { Tempo médio de } \\
\text { espera na fila }\end{array}$ \\
\hline Normal & 130 & $00: 02: 30$ & $00: 12: 00$ \\
Preferencial & 72 & $00: 04: 20$ & $00: 25: 00$ \\
\hline
\end{tabular}


Comparando o resultado dos dois tipos de fila, percebe-se que há diferença na quantidade de clientes que são atendidos, de 58 clientes. Contudo, o tempo em que o usuário está sendo atendido e o tempo que ele espera, é desfavorável para o atendimento preferencial. No tempo médio, há uma diferença de 1 minuto e 50 segundos entre os dois tipos de atendimento. Tanto no tempo médio de espera na fila quanto no de atendimento o tempo referente a clientes preferenciais é cerca do dobro dos demais clientes. Sendo assim, mesmo a população para atendimento no caixa preferencial sendo menor, devido à duração do atendimento, a espera é maior.

A seguir, o Quadro 3 demonstra os parâmetros da simulação.

\begin{tabular}{|c|c|c|c|}
\hline Fila Normal & & Fila Preferencial & \\
\hline Atendente (s) & 1 & Atendente (s) & 1 \\
\hline Taxa de chegada $(\lambda)$ & 26 & Taxa de chegada $(\lambda)$ & 14,4 \\
\hline Taxa de atendimento $(\mu)$ & 24 & Taxa de atendimento $(\mu)$ & 13,8 \\
\hline Total de clientes no sistema & 166 & Total de clientes no sistema & 86 \\
\hline Total de clientes atendidos & 130 & Total de clientes atendidos & 72 \\
\hline Tempo médio de atendimento & $00: 02: 30$ & Tempo médio de atendimento & $00: 04: 20$ \\
\hline Tempo médio de espera na fila & $00: 12: 00$ & Tempo médio de espera na fila & $00: 25: 00$ \\
\hline
\end{tabular}

Fonte: Elaboração própria (2020)

\subsection{Simulação de melhorias do sistema de filas estudado}

A primeira proposta para gerar melhorias no processo é de aumentar o número de servidores no caixa preferencial, tendo em vista que é esse posto que está com maior tempo de espera, ultrapassando o limite permitido pela Lei Estadual no 12.264/2002.

Os resultados que seriam obtidos com o aumento da quantidade de atendentes proposto, são expostos no Quadro 4. 


\begin{tabular}{|l|l|}
\hline Fila Normal & \\
\hline Atendente $(s)$ & 1 \\
\hline Taxa de chegada $(\lambda)$ & 26 \\
\hline Taxa de atendimento $(\mu)$ & 24 \\
\hline Total de clientes no sistema & 166 \\
\hline Total de clientes atendidos & 130 \\
\hline Tempo médio de atendimento & $00: 02: 30$ \\
\hline Tempo médio de espera na fila & $00: 12: 00$ \\
\hline
\end{tabular}

\begin{tabular}{|l|l|}
\hline Fila Preferencial & \\
\hline Atendente $(\mathrm{s})$ & 2 \\
\hline Taxa de chegada $(\lambda)$ & 14,4 \\
\hline Taxa de atendimento $(\mu)$ & 13,8 \\
\hline Total de clientes no sistema & 86 \\
\hline Total de clientes atendidos & 72 \\
\hline Tempo médio de atendimento & $00: 04: 20$ \\
\hline Tempo médio de espera na fila & $00: 12: 45$ \\
\hline
\end{tabular}

Fonte: Elaboração própria (2020)

No que diz respeito ao atendimento normal, não houve alterações, pois a média obtida está dentro do que é estabelecido por lei. Cabe ressaltar, que se tratando de uma média, podem ocorrer momentos no período que ultrapassem os 15 minutos, como também, há momentos que o tempo de espera esteja aquém do tempo proposto pela lei.

Quanto ao atendimento preferencial, com a ampliação de mais um operador de caixa, estimase que o tempo médio de espera fique dentro dos 15 minutos, e o tempo médio de espera na fila de 12 minutos e 15 segundos. Para isso, é necessário um caixa para atendimento do público em geral e dois caixas para o atendimento preferencial.

A segunda proposta de melhoria seria a unificação do atendimento, ou seja, os dois operadores atenderem as duas filas, alternando entre si, na ordem de um preferencial e um normal, conforme o Quadro 5.

Quadros 5- Resultados da simulação da segunda proposta
\begin{tabular}{|l|l|} 
Fila Normal + Preferencial \\
\hline \hline Atendente $(\mathrm{s})$ & 2 \\
\hline Taxa de chegada $(\lambda)$ & 50,4 \\
\hline Taxa de atendimento $(\mu)$ & 40,4 \\
\hline Total de clientes no sistema & 252 \\
\hline Total de clientes atendidos & 202 \\
\hline Tempo médio de atendimento & $00: 01: 30$ \\
\hline Tempo médio de espera na fila & $00: 07: 12$ \\
\hline
\end{tabular}

Fonte: Elaboração própria (2020)

Como podemos identificar, a existência de uma única fila, com o atendimento alternado entre as duas demandas, respeitando a população preferencial, reduz o tempo médio de atendimento e o tempo médio de espera na fila de ambas as populações, diminuindo para 7 minutos e 12 segundos o tempo médio de espera, e para 1 minuto e 30 segundos o tempo médio de atendimento. 
Em tal proposição, não houve alteração nas instalações, permanecendo com os dois caixas, e obtendo resultado melhor do que a primeira proposta de melhoria, pois reduziu-se de 12 minutos a fila normal e 12 minutos e 45 segundos a preferencial, para 7 minutos e 12 segundos as duas filas, logo, o grau de eficiência da segunda proposta demonstra-se mais satisfatório em relação à primeira.

Destaca-se que, tendo em mente uma jornada diária de 8 horas, das quais 5 horas seriam dedicadas ao atendimento, conforme ilustrado no Quadro 4, a contratação de um novo atendente representaria um dispêndio adicional de 62,5\% do salário de um escriturário bancário sem que isso representasse a solução ótima.

Tratando-se de aplicação de sistemas melhorados baseado na aplicação das Filas, SANTOS; QUINTAL; PAIXÃO; GOMES (2015) destacam que se faz imprescindível o uso das informações coletas e a coordenação do sistema a ser implementado que permita o uso pelos agentes diretamente envolvidos no processo de tomada de decisão na instituição que possibilite a alocação adequada dos recursos humanos e materiais, de acordo com as necessidades envolvidas em vários cenários.

\section{Conclusão}

O estudo da administração da produção de serviços, utilizando da Teoria das filas, pode aperfeiçoar todo o processo de atendimento no serviço bancário, contribuindo para que as instituições financeiras tenham um diferencial no mercado, que está cada vez mais acirrado.

Neste contexto, este trabalho mostrou-se relevante devido à necessidade da otimização do atendimento das instituições bancárias, visto que há um risco de as mesmas perderem credibilidade e, consequentemente, clientes, pois esses, como afirma Slack (2013), ao esperarem em fila julgam o serviço que lhes está sendo prestado, através da percepção do tempo dispendido aguardando na fila.

Destarte, mediante aplicação de análise consignada pela teoria das filas, constatou-se que a atual forma de configuração dos canais de atendimento resultava em grande risco de extrapolação do tempo de espera legal para instituições financeiras. Logo, foi possível averiguar o desempenho do sistema de filas, além de propor melhorias com o melhor uso dos recursos e do tempo.

Conclui-se, então, que apenas reconfigurando a forma como o atendimento é realizado será possível alcançar maior satisfação dos usuários do atendimento pessoal da instituição, bem 
como a economia de custo de oportunidade relativo a cerca de dois terços do salário de um atendente padrão. Assim, abre-se uma gama de oportunidades para a realização de novos estudos sobre a temática estudada, na mesma instituição ou em outras unidades bancárias.

\section{REFERÊNCIAS}

BANCO CENTRAL DO BRASIL. Ranking das Instituições por índice de reclamações (jan e fev/2017). Site Institucional. Disponível em: 〈https://www.bcb.gov.br/ranking/index.asp〉. Acesso em: 15 mai. 2017.

BATEMAN, Robert E.; BOWDEN, Royce O.; GOGG, Thomas J.; HARRELL, Charles R.; MOTT, Jack R. A.; MONTEVECHI, José Arnaldo Barra. Simulação de sistemas: aprimorando processos de logística, serviços e manufatura. Rio de Janeiro: Elsevier, 2013.

CERVO, A. L. Metodologia científica. 5 ed. São Paulo: Pearson Prentice Hall, 2002.

CORRÊA, H. L. CORRÊA, C. A. Administração de produção e operações: manufatura e serviços: uma abordagem estratégica. 2.ed. São Paulo: Atlas, 2007.

FEBRABAN. Federação Brasileira de Bancos. (2017) Pesquisa FEBRABAN de Tecnologia Bancária 2017. São Paulo.

<https://cmsportal.febraban.org.br/Arquivos/documentos/PDF/Pesquisa\%20de\%20Tecnologia\%20Banc\%C3\%A 1ria_2017.pdf>. Acesso em: 26 jun. 2017.

FEBRABAN. Federação Brasileira de Bancos. Site Institucional. Disponível em: < https://portal.febraban.org.br/noticia/3063/pt-br/>. Acesso em: 22 jun. 2017.

GOMES, A. E. et al. Utilização da ferramenta de simulação Arena na análise do sistema de filas da estação TRENSURB em Novo Hamburgo - RS. VI Congresso Brasileiro de Engenharia de Produção. Ponta Grossa, PR: CONBREPRO, 2016.

HILLIER, Frederick S.; LIEBERMAN, Gerald J. - Introdução à Pesquisa Operacional. 8 ed. São Paulo, 2006.

LIMA, Vitor Costa de; ANHOLON, Rosley; RAMPASSO, Izabela Simon; QUELHAS, Osvaldo Luiz Gonçalves. Aplicação da teoria das filas em serviços bancários. Revista Produção Online., v. 16, n. 1, p. 210241, jan./mar. Florianópolis, 2016. Disponível em: < https://www.producaoonline.org.br/rpo/article/view/2086> Acesso em: 06 mar. 2020.

PERNAMBUCO. Lei $\mathbf{n}^{\mathbf{0}} \mathbf{1 2 . 2 6 4}$, de 18 de setembro de 2002. Dispõe sobre o atendimento ao consumidor, nos caixas das agências bancárias. Disponível em:

$<\mathrm{http}: / /$ legis.alepe.pe.gov.br/arquivoTexto.aspx?tiponorma $=1 \&$ numero $=12264 \&$ complemento $=0 \& a n o=2002 \& \mathrm{ti}$ po=>. Acesso em: 20 jun. 2017

ROESCH, S. M. A. Projeto de estágio e de pesquisa em administração, guia para estágios, trabalhos de conclusão, dissertações e estudo de caso. 3 ed. São Paulo: Atlas, 2007.

SAMPAIO, P. G. V.; OLIVEIRA, S. D. Estudo de modelagem e simulação de filas num supermercado associado à análise de cenários. XXXIII Encontro Nacional de Engenharia de Produção. Salvador, BA: ENEGEP, 2013. Disponível em:

<http://www.abepro.org.br/biblioteca/enegep2013_tn_stp_177_012_21942.pdf >. Acesso em: 30 jun. 2017.

SANTOS, Marcos dos; QUINTAL, Renato Santiago; PAIXÃO, Alexandre Camacho da; GOMES, Carlos F. Simões. Simulation of Operation of An Integrated Information for Emergency Pre-Hospital Care in Rio de Janeiro Municipality. Information Technology and Quantitative Management (ITQM 2015). Elsevier, 2015. Disponível em: 〈https://www.sciencedirect.com/science/article/pii/S1877050915015860?via\%3Dihub >. Acesso em: 06/03/2020. 
SANTOS FILHO, Diolino José; MIYAGI, Paulo Eigi; MARUYAMA, Newton. Curso de ProModel. Disponível em: < http://sites.poli.usp.br/d/pmr5008/arquivos/promodel.pdf >. Acesso em: novembro 2017.

SLACK, N.; CHAMBERS, S.; JOHNSTON, R. Administração da Produção. São Paulo: Atlas, 2002.

SLACK, N; CHAMBERS, S.; JOHNSTON, R. Administração da Produção. $2^{\mathrm{a}}$ ed. São Paulo: Atlas, 2008.

SLACK, N. Administração da produção. São Paulo: Atlas, 2013.

YIN, R. K. Estudo de caso, planejamento e métodos. 3 ed. Porto Alegre: Bookman, 2005. 